\title{
Comunidades Virtuais de Aprendizagem baseadas na Teoria de Interação Social de Piaget e suportadas por redes peer-to-peer.
}

\begin{abstract}
Letícia Silva Garcia ${ }^{1}$
Antônio Carlos da Rocha Costa*

Sergio Roberto Franco*

Resumo

Este artigo apresenta a proposta de um sistema de reputação em ambientes per-to-peer baseado na teoria da interação social de Piaget, onde os um sistema de manipulação de valores, que considera regras, valores e mecanismos de manipulação destes serve de base à construção da reputação de um indivíduo na comunidade virtual de aprendizagem.
\end{abstract}

\begin{abstract}
This paper presents a proposal for a reputation system in peer-to-peer environments based on Piaget's social interaction theory, where a system of values manipulation, which takes into account rules, values and mechanisms to manipulate these values, is the basis for the construction of an individual's reputation in the virtual learning community.
\end{abstract}

\section{Introdução}

Este trabalho situa-se na área interdisciplinar da aplicação de recursos tecnológicos à educação; mais especificamente, trata da criação de comunidades virtuais visando à aprendizagem suportadas por sistemas de computação peer-to-peer, bem como da implementação de um mecanismo de reputação aplicado a estas redes, baseado na teoria de interação social de Piaget.

Em se tratando de comunidades de aprendizagem, um dos aspectos a se considerar fortemente trata da garantia de origem e qualidade da informação disponibilizada. Sendo a computação peer-to-peer um meio descentralizado de disponibilização de informação, cuja principal característica é a criação de comunidades virtuais baseadas em interesses comuns através do compartilhamento de objetos tratando destes, faz-se necessário um estudo mais aprofundado no que diz respeito à garantia de qualidade dos objetos envolvidos no processo educativo.

$\mathrm{Na}$ computação peer-to-peer o aspecto da garantia de origem dos objetos envolvidos nos processos de troca pode ser tratado através dos mecanismos de reputação (SANCHES 2002). Mecanismos de reputação são meios de, segundo critérios próprios adequados a diferentes situações, certificar que os diferentes membros da comunidade envolvidos no processo têm identidade idônea e histórico de trocas satisfatório. Neste contexto, consolida-se a necessidade de desenvolvimento de uma solução de reputação

\footnotetext{
${ }^{1}$ Mestre em Informática, Doutoranda PPGIE - UFRGS

Coordenadora do Curso de Sistemas de Informação - Faculdade Dom Bosco de Porto Alegre

${ }^{*}$ Professor PPGIE - UFRGS

* Professor PPGIE - UFRGS

V. 2 № 1, Março, 2004
} 
especialmente adaptada para as necessidades das comunidades virtuais de aprendizagem.

Uma vez que se trata de um problema de reputação em interações sociais, buscou-se na Teoria de Interação Social de Piaget por um mecanismo que considerasse o valor das interações dos usuários enquanto realizam trocas sociais. Tal teoria define um sistema de valores, baseado em valores, regras e mecanismo de manipulação de valores (PIAGET 1973), que suporta a valoração das trocas em ambientes peer-to-peer.

Isto posto, podemos apresentar o objetivo primordial deste artigo que é a definição de um mecanismo de reputação em ambientes peer-to-peer baseado na Teoria de Interação Social de Piaget.

\section{Comunidades Virtuais de Aprendizagem baseadas em peer-to-peer}

As comunidades virtuais de aprendizagem suportadas por sistemas de computação peer-to-peer beneficiam-se das características de descentralização, anonimato e segurança.

A característica da descentralização nos traz que a propriedade e o respectivo controle de recursos deva ser responsabilidade de cada ponto e/ou de cada participante. Esta nos parece a característica mais desejável no presente trabalho, uma vez que cada uma dos componentes da rede é autônomo para a disponibilização de materiais em seu próprio computador, sem a necessidade de um gerenciamento centralizado..

Da característica do anonimato as comunidades virtuais de aprendizagem se beneficiam uma vez que, a partir desta, é possível não identificar autores, leitores e ainda servidores em que os objetos são disponibilizados. Tal característica é desejável no que diz respeito a garantia do anonimato no consumo de objetos, uma vez que o consumidor deve avaliar o objeto disponibilizado e seus objetos também são disponibilizados para que sejam avaliados. $O$ anonimato atua como apoiador na manutenção do equilíbrio social no momento que evita que a identidade do avaliador seja exposta. Por outro lado, autor e produtor devem ser identificados, uma vez que a questão autoral é utilizada como critério de avaliação na sociedade e conseqüente construção de reputação.

Os sistemas peer-to-peer compartilham suas características relacionados a segurança com os sistemas distribuídos tradicionais, como por exemplo, cadeias de confiança, esquemas de troca de chaves de sessões, encriptação e assinaturas. Outro fator relativo à segurança é a existência de sistemas de gerenciamento de reputações, onde cada um dos componentes da rede é classificado segundo sua atuação social. Tal característica é o objeto deste trabalho, através da definição de um mecanismo de reputação baseado na Teoria de Interação Social de Piaget.

\section{Breve revisão da teoria de interação social de Piaget}

A teoria interacionista de Piaget nos traz que a construção do conhecimento dá-se através da relação entre o sujeito e o objeto e que esta modifica a ambos. Superficialmente, as críticas a esta trazem que Piaget ignora a questão social, uma vez que se concentra na relação de um sujeito com um objeto de conhecimento e as implicações e mudanças que acontecem em ambos ao interagirem.

Buscando a questão social no trabalho de Piaget, nos deparamos com os Estudos Sociológicos, onde as relações sociais são sistematicamente abordadas e modeladas de tal forma a compor um conjunto de regras, valores e sinais, onde cada um destes tem uma função significativa na construção das trocas sociais. 
Neste trabalho pretendemos resgatar a questão do social em Piaget, visando a melhor definição do termo reputação. Nosso interesse principal concentra-se no estudo do conceito de valor, a partir do qual se quantificam as trocas e se constrói o conceito de reputação na sociedade. Para que possamos definir corretamente tais termos, faz-se necessária uma retomada nos conceitos e na teoria de interação social proposta por Piaget.

A primeira questão a ser considerada é que em sua Teoria de Interação Social, Piaget destaca fortemente a independência entre as regras e os valores de troca. Por regras se classificam aqueles valores que são perenes à sociedade e não dependem da temporalidade, são as condições para que a sociedade ocorra e as trocas se estabeleçam. Regras definem o contexto histórico e a permanência ou não de uma sociedade. Já por valores entende-se àquelas questões que são temporais, ou seja, variam conforme o contexto social e o momento econômico. A manutenção da sociedade depende das regras, mas não dos valores. Uma mudança nas regras representa uma mudança no status quo vigente e uma conseqüente alteração nos valores até então dominantes. As regras definem os mecanismos de manipulação dos valores.

A partir desta diferenciação podemos passar à questão da diferenciação entre valores sociais e valores econômicos. Por valor social entendem-se as questões intrínsecas ao objeto da troca, tais como sua origem, a identidade de quem o produziu e a reputação do fornecedor deste objeto. Por valor econômico entende-se a quantificação do objeto da troca. Como esta é relativa e depende fortemente do valor social, Piaget trata o valor econômico como um substrato quantificado do valor social.

O conceito de mecanismos de trocas traz que a sociedade ocorre através das trocas materiais entre os indivíduos, este é seu meio e seu fim. Intrínseca a esta questão das trocas está o conceito de valor. Para que ocorra uma troca, segundo a Teoria de Interação Social de Piaget, faz-se necessário que um componente da sociedade renuncie a um valor material seu, cedendo-o a outrem. Em troca, pode receber outro valor material, uma satisfação moral ou um reconhecimento de dívida, onde a complementação do processo de troca se dará posteriormente.

Outro ponto importante na questão deste mecanismo de troca é a da renúncia. Para que um indivíduo $\alpha$ usufrua de um bem do indivíduo $\alpha$ ' faz-se necessário que este renuncie a este bem, deixando de possuí-lo. Tal questão será contraposta quando tratarmos do conceito de redes peer-to-peer. A uma renuncia material, corresponde uma virtual, sendo que na renuncia material o indivíduo $\alpha$ fica sem o bem, tem que renunciar a ele para ceder a outrem, na renuncia virtual o indivíduo $\alpha$ fica agradecido àquele que lhe cedeu o bem, sem que este tenha renunciado, pois os objetos são compartilhados.

É necessário neste momento recorrer aos esquemas lógicos definidos nos Estudos Sociológicos, de Piaget, visando a posterior construção do esquema lógico do sistema de trocas objeto deste trabalho.

Sendo:

$\mathrm{r}_{\alpha}=\mathrm{a}$ ação (ou reação) de $\alpha$ sobre $\alpha$ '

$\mathrm{s}_{\alpha^{\prime}}=$ a satisfação de $\alpha$ gerada pela reação de r $\alpha$

$\mathrm{t}_{\alpha^{\prime}}=$ a dívida de $\alpha$ ' resultante da satisfação de s $\alpha^{\prime}$

$\mathrm{v}_{\alpha}=$ a valorização de $\alpha$ por $\alpha^{\prime}$

e o símbolo de "=" a equivalência qualitativa.

Temos equação 1 :

$\left(\mathrm{r}_{\alpha}=\mathrm{s}_{\alpha^{\prime}}\right)+\left(\mathrm{s}_{\alpha^{\prime}}=\mathrm{t}_{\alpha^{\prime}}\right)+\left(\mathrm{t}_{\alpha^{\prime}}=\mathrm{v}_{\alpha}\right)=\left(\mathrm{v}_{\alpha}=\mathrm{r}_{\alpha}\right)$

Simplificando a equação 1 , temos que:

$\left(\downarrow \mathrm{r}_{\alpha}\right)+\left(\uparrow \mathrm{s}_{\alpha^{\prime}}\right)+\left(\downarrow \mathrm{t}_{\alpha^{\prime}}\right)+\left(\uparrow \mathrm{v}_{\alpha}\right)=0$ 
Esta equação representa a hipótese do esforço dispendido pelo sujeito $\alpha$ ao realizar $r$ ser equivalente à satisfação e a correspondente renuncia virtual de $\alpha^{\prime}$. Ao realizar uma ação $\mathrm{r}$, o indivíduo $\alpha$ está gerando uma satisfação no indivíduo $\alpha$ ', que, ao ficar agradecido a $\alpha$ está contraindo com ele uma dívida equivalente ao esforço dispendido por $\alpha$ para realizar a ação $r$, ou seja, há o equilíbrio normativo entre o esforço dispendido para realizar uma ação e o agradecimento recebido em troca. Convém ressaltar que por agradecimento podemos considerar créditos futuros na solicitação de realização de ações por $\alpha$ '.

As variações desta equação, amplamente descritas na Teoria de Interação Social de Piaget, nos levam aos casos de desequilíbrio social, (i) em que a ação não gera correspondente satisfação, onde o indivíduo $\alpha$ trabalha então com "prejuízo", (ii) a ação gera satisfação maior que o esforço dispendido, onde o indivíduo $\alpha$ obtém o lucro fácil, (iii) onde a ação gera satisfação, porém não reconhecimento, não causando a renúncia moral em $\alpha^{6}$ e ainda o caso (iv) onde a renúncia moral de $\alpha^{6}$ é maior que o esforço dispendido, gerando uma dívida moral desproporcional ao esforço empregado por $\alpha$ na ação.

A questão da quantificação dos valores de $\mathrm{r} \alpha, \mathrm{s} \alpha^{\prime}, \mathrm{t} \alpha^{\prime}, \mathrm{v} \alpha$, ser subjetiva vai nos ser particularmente útil quando definirmos o sistema de trocas objeto deste trabalho, uma vez que deverão ser atribuídos valores quantificáveis a cada uma destas variáveis.

Quando a ação de $\alpha$ gera um reconhecimento por parte de $\alpha^{\prime}$, estabelece-se um direito de $\alpha^{\prime}$ sobre $\alpha$, caso contrário, é gerado um crédito. Em uma referida situação, $\alpha$ ' pode fazer valer seus direitos, ou ainda utilizar-se de seus créditos, sendo que para tal será necessário estabelecer mecanismos de coerção, uma vez que $\alpha$ ' não os reconhece como direitos. Estabelece-se aí a seguinte equação:

Equação 2:

$\left(\mathrm{v}_{\alpha}=\mathrm{t}_{\alpha^{\prime}}\right)+\left(\mathrm{t}_{\alpha^{\prime}}=\mathrm{r}_{\alpha^{\prime}}\right)+\left(\mathrm{r}_{\alpha^{\prime}}=\mathrm{s}_{\alpha}\right)=\left(\mathrm{s}_{\alpha}=\mathrm{v}_{\alpha}\right)$

Simplificando a equação 2 , temos que:

$\left(\downarrow v_{\alpha}\right)+\left(\uparrow t_{\alpha^{\prime}}\right)+\left(\downarrow r_{\alpha^{\prime}}\right)+\left(\uparrow s_{\alpha}\right)=0$

O que significa que $\alpha$ ' não só reconhece a dívida, mas como a salda com um serviço equivalente, que satisfaz de forma equivalente a $\alpha$, gerando a quitação da dívida.

A partir das equações (1) e (2), trataremos o conceito de quantificação do equilíbrio social.

$\left(\downarrow \mathrm{r}_{\alpha}\right)+\left(\uparrow \mathrm{s}_{\alpha^{\prime}}\right)+\left(\downarrow \mathrm{t}_{\alpha^{\prime}}\right)+\left(\uparrow \mathrm{v}_{\alpha}\right)=0 \rightarrow$ equação1

$\left(\downarrow \mathrm{v}_{\alpha}\right)+\left(\uparrow \mathrm{t}_{\alpha^{\prime}}\right)+\left(\downarrow \mathrm{r}_{\alpha^{\prime}}\right)+\left(\uparrow \mathrm{s}_{\alpha}\right)=0 \rightarrow$ equação 2

Se 1 e 2 ocorrem simultaneamente em um bloco, podemos aplicar o conceito de anulação dos valores de $\mathrm{v}$ e $\mathrm{t}$, ficando a seguinte equação:

$\left(\downarrow \mathrm{r}_{\alpha}\right)+\left(\uparrow \mathrm{s}_{\alpha^{\prime}}\right)+\left(\downarrow \mathrm{r}_{\alpha^{\prime}}\right)+\left(\uparrow \mathrm{s}_{\alpha}\right)=0 \rightarrow$ equação 3

A partir daí temos que $\left(r \alpha=s \alpha^{\prime}\right)=\left(\downarrow r \alpha^{\prime}=\uparrow s \alpha\right)$, logo o equilíbrio social se estabelece quando uma ação do indivíduo $\alpha$ gera uma satisfação em $\alpha$ ' que é correspondida por $\alpha$ ' com uma ação que gera em $\alpha$ uma satisfação equivalente, ou seja, há equilíbrio quando esforços e proveitos se compensam parte a parte.

\section{Definição de Mecanismo de Reputação baseado na Teoria de Interação Social de Piaget}

Ao definir um mecanismo de reputação baseado na Teoria de Interação Social de Piaget pretendemos que, ao interagir com um sistema educacional onde as diferentes realidades sociais são simuláveis, o aluno possa modificar suas atitudes de acordo com as regras sociais, visando o seu progresso pessoal e o da sociedade como um todo. 
Considerando que, para Piaget, o "conhecimento humano é essencialmente coletivo e a vida social constitui um dos fatores essenciais da formação e do crescimento dos conhecimentos pré-científicos e científicos", quebrar a barreira regional para a constituição de sociedades do conhecimento pode ser considerado um avanço significativo. O fato de a sociedade ser parametrizável nos traz a possibilidade de modelagem de diferentes culturas, onde as diferentes contribuições podem receber valor econômico e moral distintos.

Quanto à forma de valoração das contribuições individuais podemos salientar a questão das contribuições serem tratadas de acordo com perfis individuais distintos, propiciando assim a evolução de cada um dos indivíduos, visando estabelecer o equilíbrio entre o mental e o social, no sentido em que o indivíduo, tornado membro adulto desta sociedade estabelecida não teria mais como pensar fora desta socialização acabada. Nesta questão, é de especial importância a manutenção do histórico individual, para que este indivíduo social possa ter a consciência de sua caminhada no sentido deste tornar-se social.

Para que tal situação ocorra, faz-se necessário um meio parametrizável de se efetivar as trocas materiais no processo de interação social, uma vez que o processo de construção social se dá através destas. Considerando que a interação entre dois sujeitos os modifica significativamente, teremos, pois um espaço de construção coletiva onde a totalidade é constituída pelo "conjunto das relações entre os indivíduos" e não meramente pela soma das contribuições individuais.

Cada individuo é anônimo na construção coletiva, desta forma, as produções individuais são avaliadas (ou seja, agregam ou perdem valor moral) na medida que contribuem para o sucesso e o funcionamento da sociedade. A estrutura social e o funcionamento da sociedade dependem de regras estabelecidas pelo gerente desta. Neste processo, não há mais espaço para o sucesso individual e cada um dos sujeitos torna-se responsável equilíbrio da sociedade.

A grande diferença das comunidades atuais está no funcionamento do sistema de pontuação social, que pode ser dinâmico e parametrizável, possibilitando àquele que gerencia a sociedade estabelecer e modificar regras sociais. $\mathrm{O}$ sucesso ou fracasso da sociedade vai ser definido pelo entendimento e atuação de cada indivíduo conforme as regras sociais estabelecidas. Estamos propondo, então, um sistema de reputação que se classifica, de acordo com a taxionomia proposta por SANCHES 2002, como dinâmico, absoluto e misto.

Considerando as trocas que ocorrem em um ambiente peer-to-peer como sendo no modelo produtor-consumidor, definimos um ambiente que possibilite o processo educativo baseado na interação social, mediado por um coordenador. Para tal, deve ser definido um sistema de valores, composto de valores, regras e mecanismo de manipulação destes.

Um ganho importante em relação aos mecanismos de troca definidos anteriormente é que a questão da renúncia material não está presente no que diz respeito às redes peerto-peer, uma vez que o recurso é disponibilizado, mas o indivíduo que o disponibiliza não tem que abrir mão do bem para que ele seja possuído pelos demais indivíduos da sociedade.

Um indivíduo $\alpha$ disponibiliza um bem, que é utilizado por um indivíduo $\alpha$ '. Ao utilizar, o indivíduo $\alpha$ ' não consome, ou seja, não gera perda para o indivíduo $\alpha$, uma vez que não se tratam de produtos materiais, ao contrário, ao consumir, é agregado valor 
moral ao bem consumido, tornando-o mais atraente para a sociedade, mas mesmo assim, é gerada uma renúncia moral em $\alpha^{\prime}$, uma vez que este fica agradecido ao indivíduo que disponibilizou o bem. Esta renúncia moral de $\alpha^{\prime}$ caracteriza o valor moral agregado a $\alpha$, ou seja, quanto mais eu disponibilizar os meus materiais, melhor será o meu desempenho na sociedade.

A quantificação dos valores acontecerá pela avaliação dos pares, ou seja, ao acessar um objeto disponibilizado por $\alpha, \alpha^{\prime}$ deverá quantificar a qualidade deste objeto (valor econômico) e o grau de relevância deste objeto na construção de seus objetos pessoais (valor moral). A partir destes valores será constituída a reputação de cada um dos indivíduos. Com base na reputação vão se estabelecer as trocas. O processo de construção da reputação de cada um dos indivíduos ocorre da seguinte forma:

Inicialmente o coordenador da sociedade estabelece, através de um formulário de classificação de parâmetros, pesos de cada um dos valores obtidos dos usuários, definindo assim as regras de funcionamento da sociedade.

Os mecanismos de manipulação que possibilitam a definição da sociedade são:

PveP $\rightarrow$ Peso valor econômico - avaliação produtor

PvmP $\rightarrow$ Peso valor moral - avaliação produtor

PveC $\rightarrow$ Peso valor econômico-avaliação consumidor

PvmC $\rightarrow$ Peso valor moral - avaliação consumidor

PNA $\rightarrow$ Peso do numero de acessos

Os pesos são relativos e o somatório destes é 100.

Os valores a serem obtidos dos usuários são:

VveP $\rightarrow$ Valor econômico da avaliação do produtor

VvmP $\rightarrow$ Valor moral da avaliação do produtor

VveC $\rightarrow$ Valor econômico da avaliação do consumidor

VvmC $\rightarrow$ Valor moral da avaliação do consumidor

A reputação de cada um dos objetos pertencentes aos componentes da sociedade será calculada através da seguinte regra:

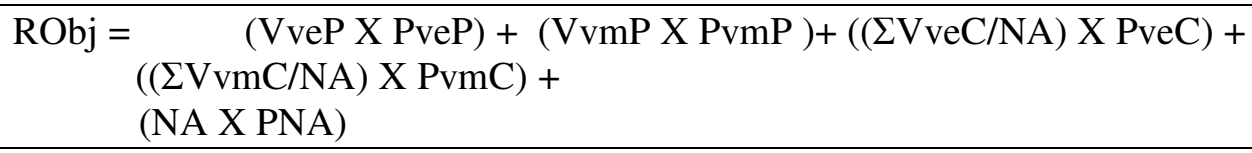

Aqui se faz necessário esclarecer o significado de cada um dos valores a serem obtidos.

VveP $\rightarrow$ Valor econômico da avaliação do produtor $\rightarrow$ qualidade que o produtor atribui ao seu objeto.

VvmP $\rightarrow$ Valor moral da avaliação do produtor $\rightarrow$

esforço dispendido pelo produtor para realizar o objeto

VveC $\rightarrow$ Valor econômico da avaliação do consumidor $\rightarrow$ qualidade que o consumidor atribui ao objeto

VvmC $\rightarrow$ Valor moral da avaliação do consumidor $\rightarrow$ aplicação que o consumidor fez do objeto na construção de seus objetos.

Para que se construa o valor da reputação de cada um dos indivíduos, faz-se necessário considerar cada um dos seus objetos, sendo que, visando minimizar o efeito de número de produções, este se dará pela média. É possível ainda ao coordenador da sociedade atribuir pesos aos diferentes objetos na construção da reputação do indivíduo 
(Pobj), sendo assim, a reputação do indivíduo na sociedade será calculada através da seguinte fórmula:

\section{REPUTAÇÃO = $\Sigma$ Robj*Pobj}

A reputação vai definir a confiança a ser depositada no indivíduo no processo de troca. Indivíduos com maior reputação serão mais procurados para fazer trocas, supondo que isso represente que os objetos que eles disponibilizam são mais confiáveis e tem melhor qualidade.

A partir destes conceitos, faz-se necessário estabelecer as condições de equilíbrio nas trocas. O equilíbrio ocorre quando o investimento realizado na produção de um objeto é equivalente à satisfação obtida pelo consumidor. Em uma sociedade em equilíbrio a diferença entre as avaliações do produtor e consumidor tende a zero. Outro fator relevante para a determinação do equilíbrio é o interesse dos membros da sociedade nos objetos disponibilizados, assim, a diferença entre os valores morais do produtor e consumidor também deve tender a zero.

Em uma sociedade equilibrada, de acordo com este conceito estabelecido, os componentes têm a real dimensão da qualidade de seus objetos disponibilizados (através do equilíbrio econômico) e estes têm real significação para todos na sociedade (equilíbrio moral). Formalizando o equilíbrio, temos então:

\section{Crédito moral social $=\Sigma \mathrm{VvmC} / \mathrm{NA}-\mathrm{VvmP}$ \\ Crédito econômico social $=\Sigma \mathrm{VveC} / \mathrm{NA}-\mathrm{VveP}$}

Crédito moral social negativo significa que os esforços na construção da sociedade não estão sendo devidamente considerados, ou que estes estão sendo realizados na direção errada.

Crédito econômico social negativo significa que as avaliações não estão corretamente dimensionadas, sendo que ou os produtores estão superestimando seus objetos ou os consumidores não estão os valorizando como deveriam. Em ambos os casos se fazem necessário a intervenção do coordenador da sociedade, através do ajuste dos pesos, visando o diagnóstico do problema e a conseqüente obtenção do equilíbrio.

EQUILÍBRIO SOCIAL $=$ Crédito moral social $=$ Crédito econômico social $=0$

\section{Conclusão}

Considerando a tendência de utilização das redes peer-to-peer nos diferentes setores de aplicação da internet, como comércio eletrônico e redes corporativas de negócios, vislumbra-se um potencial bastante amplo no que diz respeito a este recurso em aplicações educacionais. Sendo esta aplicação dotada de particularidades, faz-se necessário um estudo mais detalhado desta tecnologia visando adaptá-la a estas.

A partir disso, percebe-se o que diferencia um sistema peer-to-peer de outro é a forma como as contribuições são valorizadas no ambiente, ou seja, o mecanismo de manipulação de reputações. Este deve ser fortemente adaptável à aplicação a que se destina o sistema.

Estudos atuais contemplam aplicações da Teoria de Interação Social de Piaget no que diz respeito a modelagem de interações em ambientes educacionais. $\mathrm{O}$ conceito de valor presente nesta Teoria de Interação Social tem se mostrado bastante útil na questão da avaliação da trocas sociais ocorridas em tais ambientes. 
Convergindo estas duas questões, vislumbra-se como sendo um avanço significativo às aplicações educacionais a contribuição da proposta pela presente tese, a construção de um mecanismo de reputação para ambientes peer-to-peer baseado na Teoria de Interação Social de Piaget, uma vez que o uso de redes peer-to-peer desponta como sendo uma alternativa bastante viável pra a troca de objetos materiais de aprendizagem.

\section{Bibliografia}

COSTA, A. A Teoria Piagetiana das Trocas Sociais e sua aplicação aos ambientes de EnsinoAprendizagem. [S.1.:S.n].

COSTA, A.; DIMURO, G. Uma estrutura formal normativa para sistemas computacionais. In: VI Oficina de Inteligência Artificial. Proceedings... Pelotas, RS. Outubro 2002.

COSTA, A.; RODRIGUES, M. Using quantitative exchange values to improve the modelling of social interactions. [S.1.:S.n]

PIAGET, J. Estudos Sociológicos. Rio de Janeiro: Forense, 1973.

SANCHES, F. Utilizando mecanismos de reputação em ambientes peer-to-peer. 2002. $106 f$. Dissertação (Mestrado em Ciência da Computação) - Curso de Pós-Graduação em Ciência da Computação, UFMG, Belo Horizonte. 